\title{
DESCRIPCION DE UNA NUEVA ESPECIE DE ARTHROBRACHUS SOLIER, 1849 PARA CHILE: ARTHROBRACHUS ARUMA N. SP. (COLEOPTERA: MELYRIDAE)
}

\section{DESCRIPTION OF A NEW SPECIES OF ARTHROBRACHUS SOLIER, 1849 FROM CHILE: ARTHROBRACHUS ARUMA N. SP. (COLEOPTERA: MELYRIDAE)}

\author{
Patricia Estrada \\ Instituto de Entomología, Universidad Metropolitana de Ciencias de la Educación. \\ Casilla 147 CP 7760197 Santiago, Chile. Email: pestrada@umce.cl
}

\section{RESUMEN}

Se describe una nueva especie para el género Arthrobrachus Solier (1849), A. aruma n.sp. la que presenta apomorfías tales como antenas pectinadas y reborde lateral del pronoto mal definido, caracteres únicos en el grupo. La especie ha sido registrada sólo en la Cordillera de Doña Ana, IV Región, Chile.

Palabras claves: Coleoptera, Melyridae, Arthrobrachus, nueva especie, Chile.

\section{ABSTRACT}

A new species of the genus Arthrobrachus Solier, 1849 is described, A. aruma n.sp. The apomorphies of the new sepecies are pectinate antenna and scarcely defined lateral ridge of pronoto unique characters in the group. The species is registred from Doña Ana's range, IV Region, Chile.

Keywords: Coleoptera, Melyridae, Arthrobrachus, new species, Chile.

\section{INTRODUCCION}

Al describir el género Arthrobrachus Solier (1849) se basa, fundamentalmente, en sus antenitos y tarsitos transversos. Las 11 especies descritas para Chile (Estrada \& Solervicens 1999; Estrada 2003) presentan 3 a 6 antenitos preapicales dilatados por el costado mesal, todos los tarsitos más anchos que largos y genitalia masculina con parámeros fusionados y generalmente uniformemente esclerosados, brazos del tegmen unidos en la base originando un apodema dorsal y lóbulo medio subcilíndrico fuertemente esclerosado. La nueva especie, que se describe en este trabajo, comparte con Arthrobrachus varios de sus caracteres, especialmente lo relativo a la organización de tarsos y genitalia, por lo que se le ha adscrito a este género. Sin embargo, el nuevo taxón presenta notables apomorfías que podrían cuestionar esta clasificación. La descripción y diagnosis se basa solamente en machos, único material disponible para el estudio. 


\section{MATERIAL Y METODO}

Material

Se estudiaron 30 ejemplares obtenidos mediante trampas Barber en la cordillera de Doña Ana, provincia de Elqui. Todo el material fue donado al Instituto de Entomología de la Universidad Metropolitana de Ciencias de la Educación (IEUMCE) por el Dr. Jorge Cepeda, de la Universidad de La Serena.

\section{ESTUDIO MORFOLÓGICO}

Se analizó la morfología externa de 15 machos. Se realizaron mediciones de longitud y amplitud de cabeza, antenas, pronoto, élitros y cuerpo completo, para obtener la razón entre las dimensiones de las estructuras, las que se expresan en valores promedio con su varianza. Las mediciones se hicieron con una placa micrograduada incorporada a la lupa. Los dibujos se realizaron con cámara clara y las fotografías de barrido fueron tomadas en microscopio Jeol JSM-25- S II. La descripción sigue la nomenclatura de Kukalová-Peck \& Lawrence (1993) para las alas, de Lawrence \& Britton (1991) para los segmentos abdominales y de Sharp \& Muir (1912) para la genitalia.

\section{Resultados}

\section{Arthrobrachus aruma n. sp.}

LoCAlidad TIPO: Quebrada Pastos Largos (4.400 $4.800 \mathrm{msnm}$ ), Provincia de Elqui, Cuarta Región, Chile.

MATERIAL TIPO:

Holotipo: Macho: Cuya genitalia queda montada junto al ejemplar. Sus etiquetas consignan: "Chile, Elqui. Qda. Pastos Largos, Minera El Indio, dic 92ene 93. H. Vásquez" y "Ex. Trampas Barber"

Paratipos: Se designa una serie de 10 ejemplares, todos machos con los mismos datos del holotipo.

DePósito del MATERIAL TIPO:

- Holotipo y una serie de 5 paratipos quedan depositados en las colecciones del Museo Nacional de Historia Natural de Santiago.

- Una serie de 5 paratipos queda depositada en las colecciones del Instituto de Entomología de la Universidad Metropolitana de Ciencias de la Educación.
ETIMOLOGÍA:

aruma : noche, en lengua Aymará. Nombre que alude al período del día en que, seguramente, por el tipo de antena que presentan, están activos los individuos de esta especie.

\section{DiAgnosis}

Antenas de los machos pectinadas, con 8 antenitos prolongados por el costado mesal, largas, casi la mitad de la longitud del cuerpo (Fig. 1). Reborde lateral del pronoto mal definido (Figs.3, 4). Epipleura ancha, de amplitud constante, angostándose en la porción distal densamente pilosa en toda su extensión y dentada por el borde externo en la porción preapical. VI ventrito con amplia concavidad central en los bordes basal y distal, que lo divide en dos placas transversas. Parámeros fusionados, uniformemente esclerosados, con ápice amplio, levemente escotada al centro. Lámina del tegmen débilmente esclerosada y frecuentemente reducida a la base. Brazos del tegmen forman reborde en la base de los parámeros, convergen en la base formando un corto apodema, suavemente curvado hacia dorsal (Fig.7 a, b, c). Lóbulo medio con abertura apical, cerrada por dos láminas subtriangulares laterales y una pieza subcilíndrica central. Cuerpo levemente curvado. Apodema oval (Fig. 7 d, e, f).

\section{DESCRIPCIÓN:}

Cuerpo moderadamente alargado, $2,29( \pm 0,14)$ veces más largo que ancho, de lados subparalelos, 3,3 $\mathrm{mm}$ de longitud total promedio (rango: $2,9-3,5$ ) y $1,44 \mathrm{~mm}$ de ancho máximo promedio (rango 1,3$1,6)$. Puntuación uniforme, densa, gruesa en cabeza, pronoto y más fina y raleada en esternos torácicos y abdominales; élitros con puntos densos, amplios, de fondo membranoso. Pilosidad densa, clara, larga e inclinada, cubriendo casi la totalidad del cuerpo, más abundante en el dorso que en la región esternal y patas. Escasos pelos gruesos, claros y erectos en cabeza, pronoto y élitros. Cuerpo de color testáceo oscuro.

\section{CABEZA:}

incluidos los ojos, levemente más angosta que el pronoto $(0,859 \pm 0,072)$. Antenas pectinadas, 8 antenitos distales prolongados por el costado mesal, largas, constituyen más del doble de la longitud del pronoto $(2,34 \pm 0,181)$ y casi la mitad de la longitud total del cuerpo $(0,54 \pm 0,04)$ 
(Fig.1). Todos los antenitos dilatados con una depresión subcircular provista de sensillas (Fig. 2).

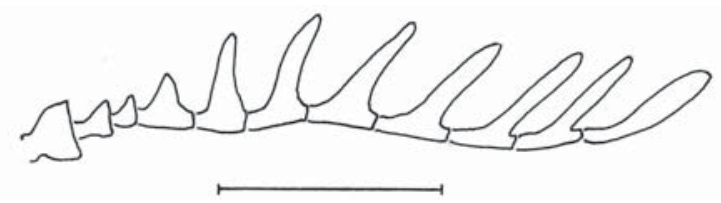

Figura 1. Antena de Arthrobrachus aruma, macho. Escala : $0,5 \mathrm{~mm}$.

Figure 1. Antenna of Arthrobrachus aruma, male. Scale: $0.5 \mathrm{~mm}$.

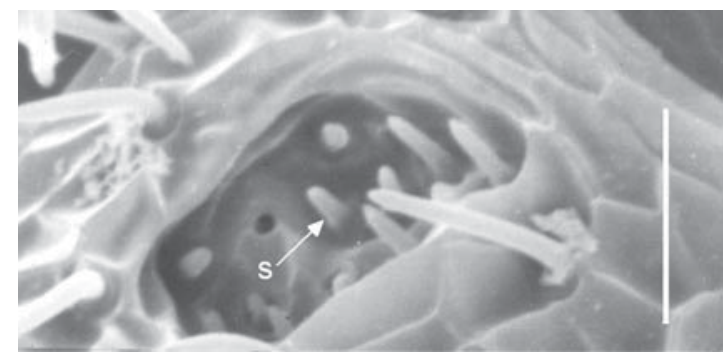

Figura 2. Detalle antena, depresión subcircular en la base del antenito; S: sensilla. Escala: 0,01 mm.

FiguRE 2. Detail of antenna, subcircular depression in the base of the antennomere; S: sensilla. Scale: $0.01 \mathrm{~mm}$.

TÓRAX:

Pronoto transverso, longitud pronotal casi la mitad de su amplitud máxima $(0,67 \pm 0,05)$, fuertemente convexo en sentido transversal; casi plano en sentido anteroposterior. Más angosto que la región humeral $(0,79 \pm 0,02)$, rebordes basal y distal levemente marcados, con leve escotadura en la línea media del reborde basal. Costados curvos, reborde lateral difuso, levemente marcado por la puntuación (Fig. 3 ); fuerte puntuación continua desde el disco al hipómero; borde lateral inexistente (Fig. 4). Elitros $1,71( \pm 0,10)$ veces más largos que anchos, costados subparalelos, redondeados en la porción media distal. Superficie moderadamente convexa en sentido transversal y suavemente descendente hacia el ápice, sin protuberancia preapical; borde marginal lateral poco marcado. Epipleura ancha de amplitud constante, angostándose en la porción distal; densamente pilosa en toda su extensión, con pelos finos, claros, inclinados, borde externo dentado en el tercio distal. Alas metatorácicas con celda radial esclerosada, apenas esbozándose r4; RP unida a RP2; rp-mp2 incompleta, a veces muy corta o inaparente (Fig. 5). Esternos y patas con abundante pilosidad clara e inclinada. Metasterno moderadamente convexo.

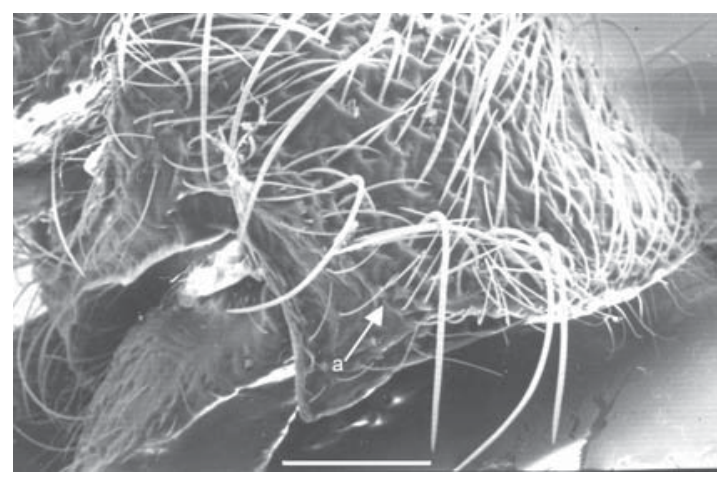

FIgURA 3. Detalle pronoto de Arthrobrachus aruma; a: puntuación que marca levemente el reborde lateral; $\mathbf{b}$ : reborde basal. Escala $0,1 \mathrm{~mm}$.

FIgURE 3. Detail of the pronotum of Arthrobrachus aruma; a: punctuation that marks lightly the lateral ridge; $\mathbf{b}$ : basal ridge. Scale: $0.01 \mathrm{~mm}$.

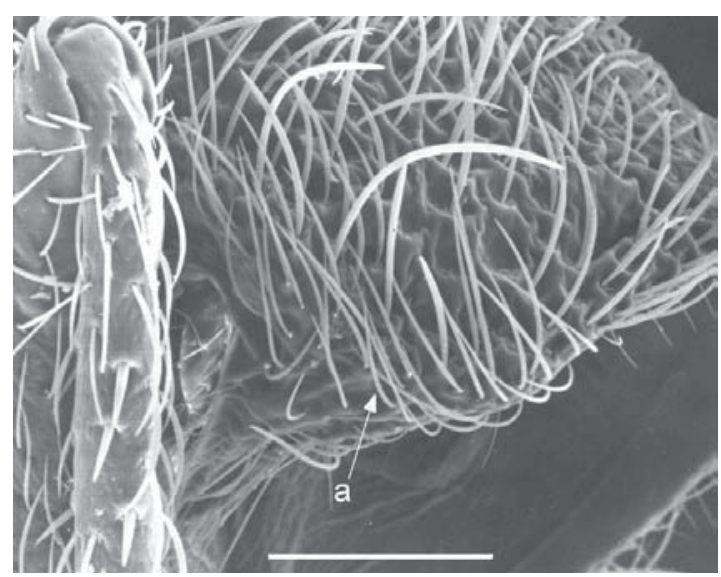

Figura 4. Vista lateral pronoto de Arthrobrachus aruma, a:puntuación desde el disco a la proyección hipomeral; b:borde basal. Escala: 0,1 mm.

FIGURE 4. Lateral view pronoto of Arthrobrachus aruma, a: basal punctuation between the disc and the hipomeral projection; b: basal ridge. Scale: $0.01 \mathrm{~mm}$. 


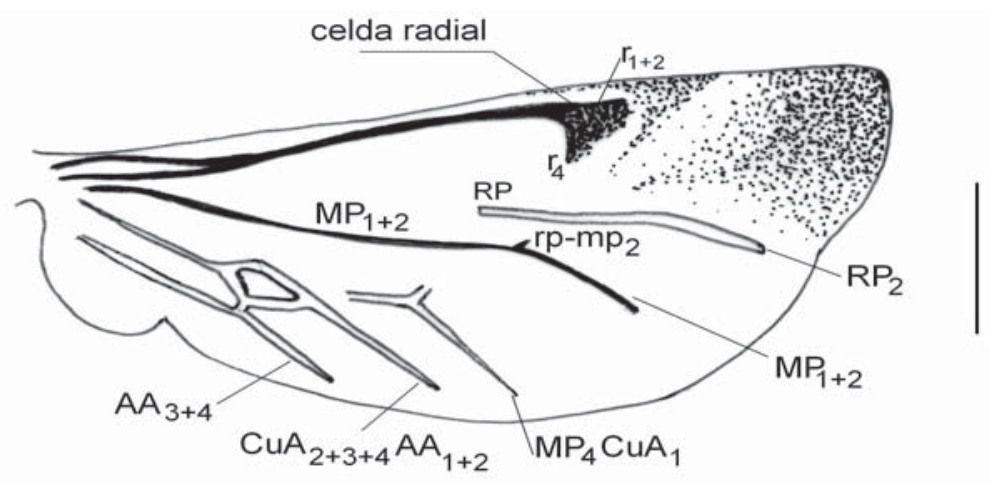

Figura 5. Ala metatorácica de Arthrobrachus aruma. Escala: 0,5 mm.

FiguRE 5. Metathoracic wing of Arthrobrachus aruma. Scale: $0.5 \mathrm{~mm}$.

ABDomen: Ventritos con abundante pilosidad clara, fina e inclinada, raleada puntuación gruesa, poco profunda. Machos: $V$ ventrito con margen distal levemente escotado en la línea media (Fig. 6c). VI ventrito formado por una placa subrectangular, borde distal y basal con amplia concavidad en la porción media que lo divide (Fig. 6 b). Ultimo tergo subcuadrado, ángulos distales redondeados, leve escotadura central en el borde distal (Fig. 6d). IX segmento: tergo esclerosado, angosto, brazos del esterno angostos, unidos por una membrana débilmente esclerosada; apodema fino, corto (Fig. 6a).

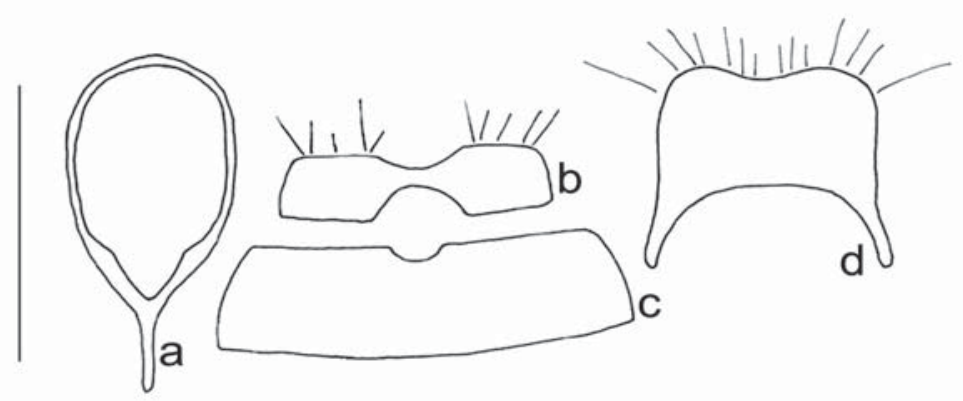

Figura 6. Segmentos terminales del abdomen de Arthrobrachus aruma, a: IX segmento; b: VI ventrito; c: V ventrito; d: último tergo. Escala :0,5 mm.

FIgURE 6. Terminal segments of the abdomen of Arthrobrachus aruma, a: IX segment; b: VI ventrite; c: V ventrite; d: last tergum. Scale : $0.5 \mathrm{~mm}$.

Genitalia: Macho: parámeros fusionados, completamente esclerosados, cortos, subplanos, ápice ancho, con leve escotadura en la línea media, bordes laterales doblados hacia mesal, fusionados en la porción media distal en ángulo obtuso con el tegmen. Tegmen con reborde dorsal completo, separado de la base de los parámeros; brazos laterales, finos, convergentes hacia la base, unidos en el extremo, formando un corto apodema, éste, suavemente curvado hacia dor- sal. Lámina del tegmen débilmente esclerosada, plana, a veces apenas insinuada en la base (Fig. 7 a,b,c). Lóbulo medio: cuerpo subcilíndrico, levemente curvado, costados subparalelos, convergentes hacia distal; ápice abierto, con dos láminas laterales subtriangulares, convergentes y una pieza subcilíndrica central; apodema oval, costados doblados hacia mesal, abertura ventral amplia proyectada hacia la base del cuerpo del lóbulo medio (Fig. 7 d,e,f). 
Gayana 69(2), 2005

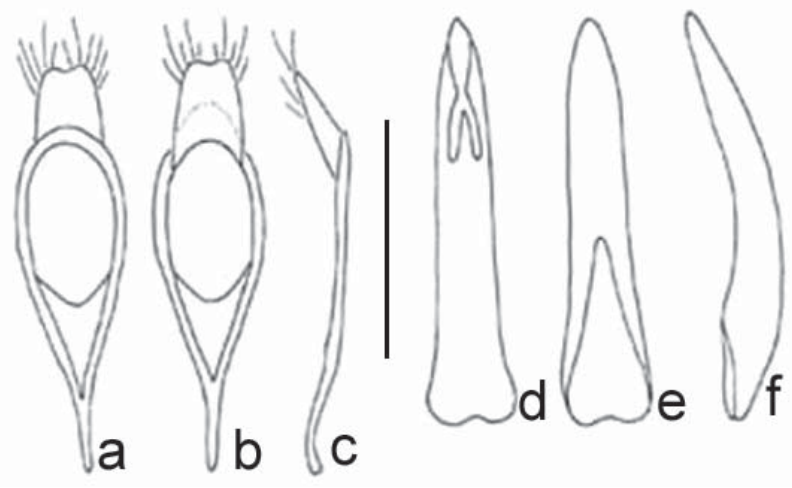

Figura 7. Genitalia masculina de Arthrobrachus aruma. Tegmen y parámeros, a: vista dorsal; b: vista ventral; c: vista lateral. Lóbulo medio: d: vista dorsal; e: vista ventral; f: vista lateral. Escala 0,5 mm.

Figure 7. Male genitalia of Arthrobrachus aruma. Tegmen and parameres; a: dorsal view; b: ventral view; c: lateral view. Median lobe: d: dorsal view; e: ventral view; f: lateral view. Scale: $0.5 \mathrm{~mm}$.

NOTAS BIOLÓGICAS:

Esta especie ha sido colectada solamente a través de trampas Barber, entre diciembre y enero; se desconocen datos sobre hospederos y de ciclo de vida.

DISTRIBUCIÓN:

Sólo se ha registrado en la provincia de Elqui en zonas de altura entre 3.000 y $4.800 \mathrm{msnm}$.

Registro de Localidades: Chile: Provincia de Elqui: Quebrada Pastos Largos (4.400-4.800 msnm), diciembre 1992-enero 1993. 29 ej. (IEUMCE); Quebrada La Mula (3.100 msnm), enero 1993. 1 ej. (IEUMCE).

\section{DISCUSION}

La organización del edeago de Artrhobrachus aruma n.sp. corresponde con la distintiva del género y muy semejante con la de Arthrobrachus limbatus y A. latefasciatus (Estrada \& Solervicens 1999). Sin embargo, A. aruma difiere notablemente por presentar antenas pectinadas y el reborde lateral del pronoto mal definido, caracteres únicos en la tribu Arthrobrachini (Majer 1987). Considerando que además de los caracteres genitales la organización de antenas y tarsos con artejos cortos y anchos son diagnósticos para Arthrobrachus (Solier 1849) y que el reborde lateral del pronoto se manifiesta claramente definido a nivel de familia (Majer 1987,1994) es que se requiere un análisis filogenético del grupo para aclarar las relaciones de parentesco intragenéricas y eventualmente separar en grupos de especies o crear un nuevo taxón donde adscribir a esta nueva especie y sus más cercanas.

\section{AGRADECIMIENTOS}

Al Prof. Jaime Solervicens A., del Instituto de Entomología de la Universidad Metropolitana de Ciencias de la Educación, por las sugerencias al manuscrito y por la confección de los dibujos. Al Dr. Jorge Cepeda y al Sr. Hernán Vásquez, de la Universidad de La Serena, por la donación del material estudiado. Al Prof. Jaime Pizarro A., de la Universidad de La Serena, por la información sobre los datos de colecta. A la Prof. Ximena Vergés, del Laboratorio de Microscopia Electrónica de la P. Universidad Católica de Chile, por su cálida asistencia en la toma de fotografías de barrido. A la Dirección de Investigación de la Universidad Metropolitana de Ciencias de la Educación por otorgar los fondos para el financiamiento de la publicación. 


\section{BIBLIOGRAFIA}

Estrada, P. 2003. Nueva especie chilena del género Arthrobrachus Solier, 1849: Descripción de Arthrobrachus epumenki n.sp. (Coleoptera : Melyridae). Acta Ent. Chilena 27:51-55.

Estrada, P. \& J. Solervicens. 1999. Revisión taxonómica de las especies chilenas del género Arthrobrachus Solier, 1849 (Coleoptera : Melyridae). Acta Ent. Chilena, 23:41-81.

Kukalová-Peck, J. \& J. F. Lawrence. 1993. Evolution of the hind wing in Coleoptera. The Canadian Entomologist, 125:181-258.

MAJER, K. 1987. Comparative morphology and proposed major taxonomy of the family Melyridae (Insecta:Coleoptera). Plskie Pismo Entomologiczne, 56:719-859.

Majer, K. 1994 A review of the classification of the Melyridae and related families (Coleoptera : Cleroidae). Ent. Bas., 17:319-390.

Lawrence, J.F. \& E. B. Britton. 1991. Coleoptera. En: The insects of Australia, CSIRO, Melbourne University Press. $2^{\mathrm{a}}$ ed. Vol. II. $1137 \mathrm{pp.}$

Sharp, D. \& F. Muir. 1912. The comparative anatomy of the male genital tube in Coleoptera. Trans. Ent. Soc. London. 1912:477-642.

Solier, A. 1849. Orden III. Coleópteros. En Gay, Historia Física y Política de Chile, Vol. 4:414-418.

Fecha de recepción: 20/12/04

Fecha de aceptación: 10/05/05 